\title{
Passive, active and intra-active (self) touch ${ }^{\text {is }}$
}

\author{
S.J. Bolanowski ${ }^{\text {a,b, }}$, R.T. Verrillo ${ }^{\text {a }}$, Francis McGlone ${ }^{\text {b }}$ \\ ${ }^{a}$ Institute for Sensory Research and Department of Bioengineering and Neuroscience, Syracuse University, Syracuse, NY 13244, USA \\ ${ }^{\mathrm{b}}$ Port Sunlight Laboratory, Unilever Research, Wirral CH63 3JW, UK
}

Received 5 May 2003; received in revised form 6 May 2003; accepted 6 May 2003

\begin{abstract}
A series of experiments are described in which magnitude estimates of the perceived size of steel balls were made when the balls were actively rolled between the fingertip and several other body sites (thumb, thenar eminence, forearm). This movement, called scripting, involves actively moving an object by a touching surface over another surface of the body which is passively being touched. We define this active/passive activity as "intra-active touch" and the results show that the perceptual size of the balls is dependent upon the body part passively being activated. An additional series of experiments decoupled the actively generated and passively received tactile information by having subjects either perform the scripting on another individual's body site or by having the other individual roll the balls on the subject's various sites. The latter experiments showed that the passive body can contribute to the overall impression of the size of the balls, but only when the intra-active touching involved the glabrous skin of the hands. Intra-active touch between the active finger and the passively touched hairy skin of the forearm showed no effect of the touched surface on the perceived size of the balls. The results suggest that the mechanisms of intra-active touch are different when glabrous skin activates glabrous skin than when glabrous skin activates hairy skin.
\end{abstract}

(C) 2003 Elsevier B.V. All rights reserved.

Keywords: Active touch; Psychophysics; Somatosensation; Tactile perception

\section{Introduction}

It is appropriate, almost one hundred years later, to celebrate both the persona of David Katz and the arrival of the millennium in this city of Stockholm. The millennium will, of course, pass, but the insights and contributions Professor Katz will no doubt last far into future millennia. It is thus fitting to speak of ideas first documented by Katz, and to acknowledge others who probed the mysteries of somatosensation. For example, it was Katz [7] who first discussed the concepts of passive and active touch and work on these aspects of touch continue. And it was Katz [7] who first spoke of "dual-touch," the somatosensory system stimulating itself. This is quite unlike any of the other sensory systems: we and all living creatures do this almost continuously and unconsciously in such activities as grooming, cleansing and in the interest of beauty.

\footnotetext{
This paper should have appeared in the Special Issue of BBR on "Brain Mechanisms of Tactile Perception," Vol. 135, No. 1/2, 2002.

* Corresponding author. Tel.: +1-315-443-4164; fax: +1-315-443-1184.

E-mail address: sandy_bolanowski@isr.syr.edu (S.J. Bolanowski).
}

Gibson [4,5], too, mused about the differences between active and passive touch, although he did not discuss dual-touch. He pointed out the differences in perception that occur in passive touch compared to active touch. In active touch, a person touching someone or something else, produces an impression of that which is touched. To use Katz's [6] terminology, in active touch the objective pole, "what's out there," prevails. Passive touch, in which items touch an individual evoke a distinctly different subjective percept, that of an internal sensation confined not to the environment but to oneself: riveting, with a sense of immediacy.

In the work that is reported herein, the notion of dual-touch or what we will call "intra-active" touch is explored. In performing such actions, what is actually felt: the body part doing the touching (active touch) or the body part being touched (passive touch)? We can reflectively appreciate that there is a profound difference between someone touching oneself (intra-active touch), touching someone else (active touch) and being touched by another (passive). Whereas Weber ([9], cited in [6]) and Katz [6] have discussed the issue of intra-active touch from a phenomenological standpoint, almost nothing is known about its underlying processes and mechanisms. The experiments 
reported here are the first attempt since Katz's earlier studies to address the matter of intra-active touch psychophysically and have previously been published [2].

\section{Materials and methods}

The stimuli consisted of nine chrome-steel ball bearings ranging in surface area from 0.5 to $127 \mathrm{~mm}^{2}$ (diameter, 0.4-6.4 mm). Six subjects made absolute magnitude estimates of the "sizes" of the steel balls under a variety of conditions in which the balls were rolled between the skin surfaces of two body sites, as well as on a skin-like foam surface that acted as a control. The term "size" was not explicitly defined by the experimenter and thus the subjects could use any subjective impression of size that they felt comfortable with.

The subjects were instructed in the absolute magnitudeestimation procedure (see [1,3]). During the experiments, the different sized balls were placed by the experimenter between the two surfaces to be used. Subjects were blindfolded and were allowed to rotate the balls between the two body parts for as long (usually about $5 \mathrm{~s}$ ) as they wished, using a circular-like movement (scripting). When satisfied that they could estimate the ball's size, they called out a number indicative of their subjective impression of the size of the ball. In each experimental session there were three blocks of trials. Each block contained all of the nine balls in random order. Individual estimates of the size of the balls in each session were computed by taking the geometric mean of the magnitude estimates of size obtained during the second and third blocks within the session. Group geometric means were obtained from the normalized individual mean estimates (see [2] for normalization procedure).

\section{Results}

In the first series of experiments the balls were placed between the glabrous skin of the subjects' distal pad of the right forefinger and left thumb. Because the balls were rolled between the finger and thumb, the task could be thought of as intra-active touch (self-stimulating-self). The results are shown in Fig. 1 (open circles). Plotted on double-logarithmic axes are the subjective sizes of the balls as a function of the ball's surface area. Surface area was chosen as the independent variable based on post-testing interviews of the subjects who reported this dimension as the most salient (see Section 4). The data points, follow a straight line on the $\log -\log$ plot indicating that the relationship between subjective size and surface is a power-function. A regression analysis of these data yield a power-function exponent of 0.88 $(r=0.998)$. The second set of experiments was designed to determine how the skin of the passive body site (i.e. the skin being touched) contributes to the overall impression of size. One way to test this is to use information concerning

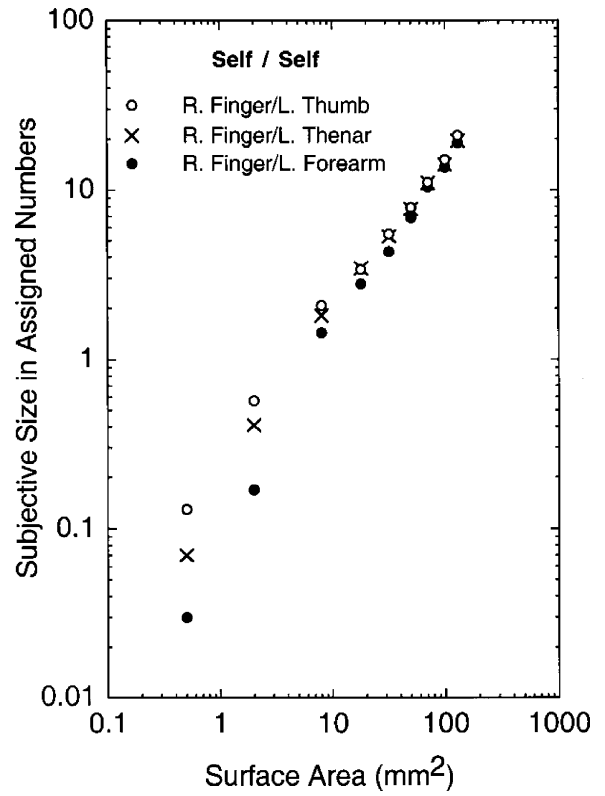

Fig. 1. The mean subjective estimates of size of the balls produced by scripting them between the right forefinger and the left thumb $(\bigcirc)$ left thenar eminence $(x)$ or the left volar forearm $(\mathbf{O})$.

the different characteristics of the different skin types of the (see, for example [6]). Specifically, the glabrous skin of the fingers is known to have a higher innervation density and greater sensitivity than the palm, and the hairy skin of the volar forearm is known to have not only differences in innervation density and sensitivity, but receptor type as well. To determine if the passive body part contributes to the overall impression of size, the subjects estimated the ball's sizes by rolling them between the right forefinger and the left thenar eminence and again between the right forefinger and the left volar forearm. The results of these two conditions (again self/self) are compared in Fig. 1 along with the results of the right forefinger/left thumb condition.

The figure illustrates that for the smaller ball sizes, the subjective estimates of size seem different in the three conditions. There is a marginally significant difference between the right finger/left thumb and the right finger/left thenar conditions (ANOVA, $P=0.053$ ). However, there is a highly significant difference between the results obtained in the right finger/left forearm and the right finger/left thumb $(P=$ $0.0001)$ conditions, as well as in the right finger/left forearm and the right finger/left thenar $(P=0.007)$ conditions. These results suggest that the passive body part engaged in intra-active touch may contribute differentially to sensation.

The next set of experiments was designed to determine whether both the body part doing the touching and the body part being touched contribute to the overall impression of size. In these experiments we sought to decouple the active and passive components of intra-active touch by using two individuals in the estimation of ball size. First, the subject rolled the balls between their own right forefinger and the thenar eminence of another person (a "standard"). 


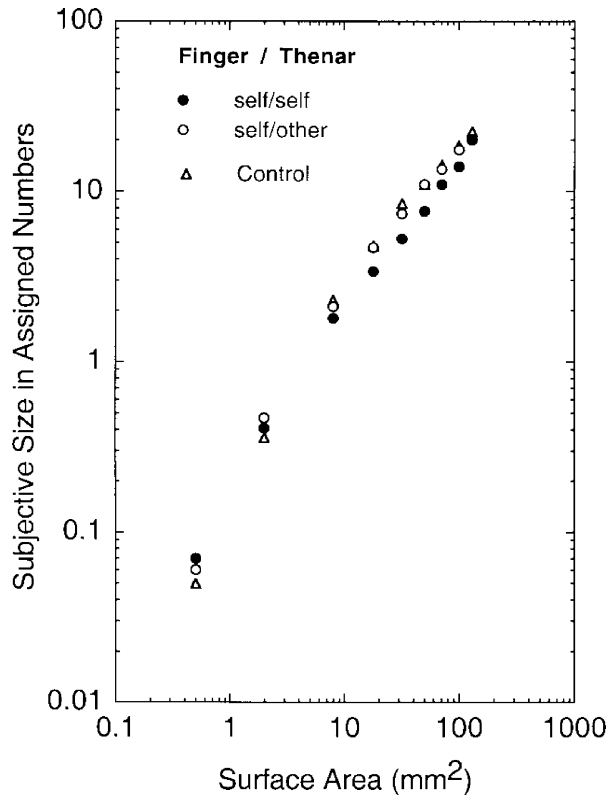

Fig. 2. The mean subjective estimates of the size of the balls produced by having the subject roll the balls on their own thenar eminences (self/self), another person's thenar eminence (self/other) and when the subject rolled the balls on a skin-like, foam surface (control).

In this condition (self/other), the subjective size of the ball could be estimated based on information arising solely from the actively touching forefinger, although the stimulus conditions were otherwise the same. In the second condition, the subject rolled the balls on a neutral foam surface that felt like the glabrous skin of the thenar eminence. As in the first condition, the subjective size of the ball could be estimated based on information arising solely from the actively touching forefinger. In the third condition, the forefinger of another person (the "standard") rolled the balls on the thenar eminence of the subject. In this condition (other/self) only the passive activation of the thenar eminence could signal the sensation magnitude of the ball's size. The results of these experiments are shown in Figs. 2 and 3 .

Fig. 2 shows the results obtained for the condition in which the subject rolled the balls on the other person's thenar eminence (self/other) compared to the condition in which the subject rolled the balls on their own thenar eminence (self/self). The results are significantly different $(P=0.001)$. That is, the self/self condition produced estimates of the ball's sizes that were less than those obtained in the self/other. This indicates that the body site being touched can influence perception during intra-active touch, in this instance perhaps via some "subtractive" mechanism. A similar result was obtained when the subjects rolled the balls on a neutral foam surface. The latter results are also shown in Fig. 2 (control). There is no significant difference between the control condition and the results obtained in the self/other condition $(P=0.15)$, although when the control condition was compared to

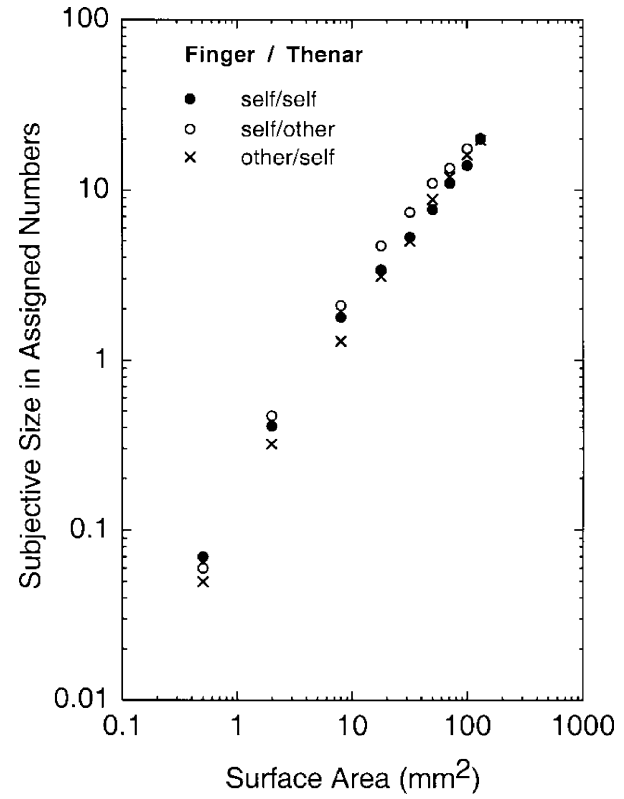

Fig. 3. The mean subjective estimates of the size of the balls produced by having the subject roll the balls on their own thenar eminence (self/self), on another person's thenar eminence (self/other) and by having the standard person roll the balls on the subject's thenar eminence.

the self/self case the results were highly significantly $(P=0.001)$.

Fig. 3 shows the results of the condition in which the "standard" forefinger rolled the balls on the subject's thenar eminence (other/self) and the results are compared with the self/self and self/other conditions replotted from Fig. 2. There is no statistically significant difference between the self/self and the other/self conditions $(P=0.47)$ indicating that in the absence of active touch a passively touched body part may be totally capable of signaling certain stimulus dimensions in a manner identical to that achieved by intra-active touching. It is as if the actively moved body part, at least given the conditions used here, contributes little to the overall impression of ball size. Furthermore, the finding that the subjective impression of ball size is no different whether passively applied to the thenar eminence or applied using intra-active touch indicates that differences in sensitivity between the fingertip and the thenar eminence probably plays no significant role in the task used here. The results shown in Figs. 2 and 3 indicate that during intra-active touch, both the passive and active body parts can contribute to overall tactile perceptions, but in the absence of an active body movement the subjective impressions of externally applied stimuli may be well described solely by the passively receiving site. The last set of experiments was designed to determine under what conditions the active and the passive body parts contribute to the overall impression of stimulus size during intra-active touch. As in the fingertip/thenar conditions discussed in relation to Figs. 2 and 3, the last experiments were designed to decouple the active and passive components of the intra-active touch process 


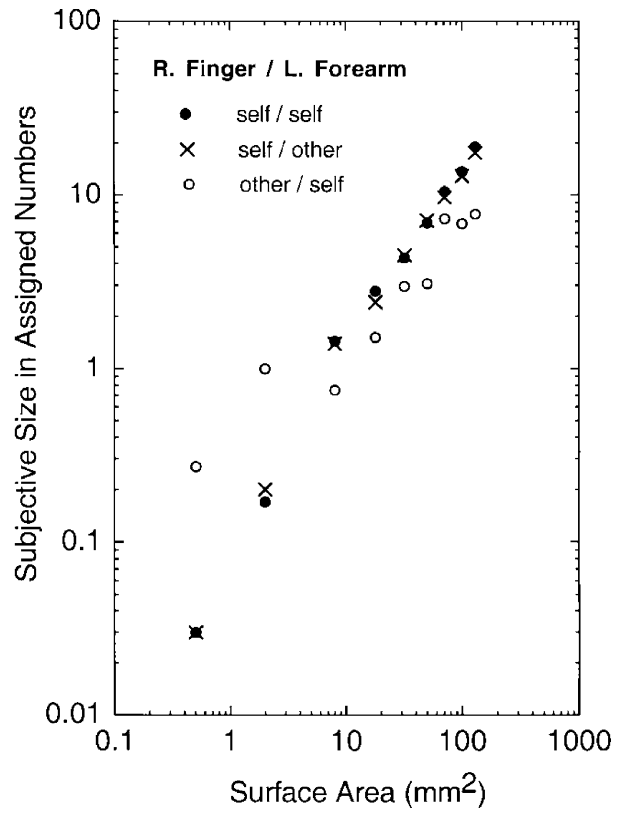

Fig. 4. The mean subjective estimates of the size of the balls produced by having the subject roll the balls on their own volar forearm (self/self), on another person's volar forearm (self/other) and by having the standard person roll the balls on the subject's forearm.

by using two individuals at a time to estimate the size of the balls. However in this case, the subject rolled the various sized balls between their own right forefinger and the "standard" volar forearm of another person (self/other). In this condition, the subjective size of the ball could be estimated only by information arising from the actively touching forefinger, with no input from the forearm. In the second condition, a "standard" forefinger rolled the balls on the volar forearm of the subject (other/self). In this condition only the passive activation of the forearm could signal the sensation magnitude of the ball's size. The results of these experiments are shown in Fig. 4 along with the results from the experiments in which the subject used their own right forefinger to roll the balls over their own left volar forearm (self/self).

It can be seen in Fig. 4, that there is no statistically significant difference ( $P=0.74)$ between the subjective impression of size produced by one touching themselves by actively rolling the ball over one's own passive body part (self/self) and the individual rolling the ball on someone else's forearm. It is as if the touched (passive) forearm has no effect on the sensation, yet we know from the results presented in Figs. 2 and 3 that the passive body also can contribute to the sensory impression of size. One possible explanation for the difference in results is that the mechanisms activated when glabrous skin intra-acts with glabrous skin are different than the mechanisms engaged when glabrous skin intra-acts with hairy skin. In the former case the touched body part seems to suppress the overall impression of size, but this does not occur when glabrous skin activates hairy skin. It is possible that the effect is somehow related to differences in sensitivity: the poorer sensitivity of the forearm may not yield enough "input" to significantly affect that arising from the forefinger.

That the fingertip/forearm conditions yield different results than the fingertip/thenar eminence condition is verified by the results obtained using the remaining experimental condition, the estimates of ball size made in response to the ball being rolled over the skin of the subjects forearm by another individual (other/self). The results, also shown in Fig. 4, reveal that although the subjects do have the capability of estimating subjective size with passive stimulation of the forearm, the relationship is quite different from that found for either of the other forefinger/forearm conditions (self/self versus other/self, $P=0.0079$; self/other versus other/self, $P=0.015)$. The smaller-sized balls are judged to be larger and the larger-sized balls are judged to be smaller than in the conditions in which the forefinger is used as the basis for the size estimates. The poor capability of discerning the size of the balls in the other/self forearm condition may be related to the lower tactile sensitivity of the forearm compared to the finger and this could explain the lack of inhibition of the balls' sizes during intra-active scripting between glabrous and hairy skin. Alternatively, the differences in glabrous skin/glabrous skin interactions and glabrous skin/hairy skin interactions may reflect differences in the ecological purpose for the two types of skin as will be described in Section 4.

\section{Discussion}

The experiments described here reveal several findings. First, both the touching and touched body part can contribute to the perceptual experience. For example, as discussed in relation to Fig. 1, different magnitude-estimation functions are obtained when the scripting involved the glabrous skin of the fingertip and either the glabrous skin of the thumb or thenar eminence than when the hairy skin of the forearm was the touched surface. This finding suggests differences in how the stimuli activate the skin or in the processing of information from hairy skin compared to glabrous skin. Hairy skin, for example, probably has a very different role than glabrous skin in deducing objects/events in the environment. The glabrous skin of the hand seems ideally suited for fine texture discrimination and is the body part upon which the concept for haptics was developed. The hairy skin of the rest of the body seems more to be involved in signaling the locus of events touching the body, with fine texture discrimination and active touching not usually performed by this region of the body.

The differences between glabrous and hairy skin being touched was further assessed in the experiments in which either the subjects touched another individual or another individual touched the subject. Again, there was a difference between glabrous skin scripting upon glabrous skin and 
glabrous skin scripting upon hairy skin. The results indicate that it is possible to judge stimuli applied either to the thenar eminence or the forearm surface and this in itself is not surprising. Nor is it surprising that actively touching an object can signal information about the object whether or not it is interposed between two body parts of the same individual. What is interesting here is that, during intra-active touch between the finger and the forearm, it is as if there is little contribution from the passive body part when the actively moving fingertip scripts objects over the passive forearm. To put this observation into a different context, as Katz [8] and Gibson [4] point out, in active touch the objective pole (environment) prevails. This is in contrast to passive touch in which the subjective pole prevails. Since we seldom, if ever, use hairy skin or body parts other than the hand to determine the shape of objects, one wonders whether the objective/subjective polar distinctions occur for hairy skin as they occur for the glabrous skin of the hand. Indeed, the touching of objects by the fingers or the hand produces a very different sensation then when the fingers or the hand are passively touched, but we have observed phenomenologically that there is no such difference when the forearm skin actively touches an object or the forearm skin is passively touched. It is as if the polar distinctions break down with hairy skin, and this may be related to the intra-active touch differences between glabrous/glabrous skin and glabrous/ hairy skin.

\section{Acknowledgements}

The authors would like to thank the subjects who participated in the experiments, the technical staff of the Institute for Sensory Research, Unilever Corporation and NIH, NIDCD (P01-000380).

\section{References}

[1] Bolanowski Jr SJ, Gescheider GA, editors. Ratio scaling of psychological magnitudes. Hillsdale, NJ: Erlbaum; 1991.

[2] Bolanowski SJ, Verrillo RT, McGlone F. Passive, active and intra-active (self) touch. Somatosens Mot Res 1999;16:304-11.

[3] Gescheider GA. Psychophysics: the basics. Hillsdale, NJ: Erlbaum; 1997.

[4] Gibson JJ. Observations on active touch. Psychol Rev 1962;69:47790.

[5] Gibson JJ. The senses as considered as perceptual systems. Boston: Houghton Mifflin; 1966.

[6] Greenspan JD, Bolanowski SJ. The psychophysics of tactile perception and its peripheral basis. In: Kruger L, editor. Handbook of perception and cognition 7: pain and touch. San Diego: Academic Press; 1997. p. 25-103.

[7] Katz D. The world of touch. Hillsdale, NJ: Erlbaum; 1989. [Krueger LE, Trans.].

[8] Katz D. Der Aufbau der Tastwelt. Zeit. für Psychologie 1925; Ergänzungsband 11.

[9] Weber EH. Der Tastinn und das Gemeingfuehl [The sense of touch and common sensibility or coenesthesia]. In: Wagner R, editor. Handwoerterb uch der Physiologie 1846;III(Pt 2):481-8. 